\title{
The sure-thing principle and the comonotonic sure-thing principle: An axiomatic analysis
}

\author{
Peter Wakker \\ Medical Decision Making Unit, University of Leiden (AZL, \# 43), P.O. Box 9600, \\ Leiden 2300 RC, Netherlands
}

Submitted October 1994; accepted January 1995

\begin{abstract}
This paper compares classical expected utility with the more general rank-dependent utility models. It shows that the difference between the sure-thing principle for preferences of expected utility and its comonotonic generalization in rank-dependent utility provides the exact demarcation between expected utility and rank-dependent models.
\end{abstract}

JEL classification: D81; C60

Keywords: Rank dependence; Nonexpected utility; Comonotonicity; Sure-thing principle; independence

\section{Introduction}

The comonotonic sure-thing principle is a necessary condition for rank-dependent utility. This paper shows, for all presently existing axiomatized versions of rank-dependent utility, that they reduce to expected utility if and only if the comonotonic sure-thing principle can be strengthened to the sure-thing principle in full force. What may be more surprising than the result itself, to most readers, is that the proof is not elementary, but rather complicated, and depends on the structural conditions of the axiomatization of rank-dependent utility that is adopted.

\footnotetext{
This paper received helpful comments from Ido Erev and Elke Weber.
} 
What may be most surprising, is that the result does not hold true for rank-dependent utility in general. This will be demonstrated by an example based on techniques from qualitative probability theory. Therefore, the above clause 'for all presently existing axiomatized versions' is essential. The example also illustrates some pitfalls in the empirical interpretation of axiomatizations.

The result of this paper can be interpreted as follows for all presently existing versions of rank-dependent utility: the generalization of rank-dependent utility theories in comparison with expected utility lies precisely in the relaxation of the sure-thing principle to the comonotonic sure-thing principle. That is, the difference between the comonotonic sure-thing principle and the sure-thing principle is the critical empirical demarcation between rank-dependent utility and expected utility.

The result of this paper has been used in an experiment by Wakker et al. (1994) to critically test the generalization that rank-dependent utility provides in comparison with expected utility. The test was done for the context of risk with given probabilities. The findings of the experiment are negative, i.e. they suggest that the rank-dependent utility model in general does not provide an empirical improvement over expected utility, and that specified forms of rank-dependent utility must be found that better capture psychological processes in human decision making. A test for the context of uncertainty is being prepared (Fennema and Wakker, 1995).

Finally, it is emphasized that the sure-thing principle and its comonotonic weakening serve to distinguish between expected utility and rank-dependent utility, but do not have an identical role in distinguishing between these models and other models. Thus, it is well-known that the sure-thing principle in isolation does not characterize expected utility. Chew and Wakker (1993) characterize the comonotonic sure-thing principle and show that it does not imply rank-dependent utility.

\section{Definitions and the main result}

$(S, \Sigma)$ denotes the state space endowed with a sigma-algebra. Elements of $\Sigma$ are events. $(\mathscr{C}, \Delta)$ is the outcome space endowed with a sigma-algebra. $\mathscr{F}$ denotes the set of (simple) gambles, i.e. measurable functions from $S$ to $\mathscr{E}$ that take only finitely many values. The term gamble is chosen here instead of 'act' to emphasize that the results of this paper hold as well for decision under risk as for decision under uncertainty (these models are defined below).

The theorems of this paper, when formulated only for the set $\mathscr{F}$ of simple gambles, immediately imply the same results for larger sets, hence the analysis is restricted to simple gambles. By $\succeq$ we denote a binary, 'preference,' relation on $\mathscr{F}$. We assume throughout that $\succeq$ is a weak order, i.e. it is complete (for all gambles $f, g, f \succeq g$ or $g \succeq f$ ) and transitive. Notations $\succ, \preccurlyeq, \prec$, and $\sim$ are as usual. $\succeq$ is trivial if $f \succeq g$ for all $f, g$. Preferences on $\mathscr{E}$ are derived from preferences over constant gambles and are denoted by the same symbols. An event 
is null w.r.t. a set $E \subset \mathscr{F}$ of gambles if all gambles $f, g \in E$ that agree outside the event, are indifferent. The opposite of null is non-null. If $E=\mathscr{F}$ then 'on $E$ ' is usually omitted.

$W$ is a capacity if $W: \Sigma \rightarrow[0,1], W(\emptyset)=0, W(S)=1$, and $A \supset B \Rightarrow W(A) \geq$ $W(B)$. For a function $\varphi: S \rightarrow \mathbb{R}$, the Choquet integral of $\varphi$ with respect to a capacity $W$ is defined as

$$
\int_{\mathbb{R}^{+}} W(\{s \in S: \varphi(s) \geq \tau\}) \mathrm{d} \tau+\int_{\mathbb{R}_{-}}[W(\{s \in S: \varphi(s) \geq \tau\})-1] \mathrm{d} \tau .
$$

A function $V$ represents $\geq$ if $V: \mathscr{F} \rightarrow \mathbb{R}$ and $f \geq g \odot V(f) \geq V(g)$. Rank-dependent utility (RDU) holds if there exist a capacity $W$ and a utility function $U$ : $\mathscr{C} \rightarrow \mathbb{R}$ such that $\succeq$ is represented by

$$
\begin{aligned}
f \mapsto & \int_{\mathbb{R}^{+}} W(\{s \in S: U(f(s)) \geq \tau\}) \mathrm{d} \tau \\
& \quad+\int_{\mathbb{R}_{-}}[W(\{s \in S: U(f(s)) \geq \tau\})-1] \mathrm{d} \tau .
\end{aligned}
$$

The above value, the Choquet integral of $U \circ f$ over $S$ with respect to $W$,is the rank-dependent utility of $f$. Expected utility $(E U)$ is the special case where $W$ is additive. Under EU, $\succeq$ satisfies the sure-thing principle, i.e. $f \succeq g \Leftrightarrow f^{\prime} \succeq g^{\prime}$ whenever $S$ can be partitioned into events $I$ and $R$ such that $f=g$ and $f^{\prime}=g^{\prime}$ on $I$, and $f=f^{\prime}$ and $g=g^{\prime}$ on $R$.

A set of gambles $F$ is comonotonic if there do not exist $f, g \in F$ and states $s$, $t$ such that $f(s) \succ f(t)$ and $g(s) \prec g(t)$. Under RDU, $\succeq$ satisfies the comonotonic sure-thing principle, i.e. the sure-thing principle holds within comonotonic sets of gambles.

Decision under risk is the special case where a probability measure $P$ is given on $(S, \Sigma)$, and gambles that generate the same probability distribution over $\mathscr{C}$ are indifferent. In the literature, gambles are then identified with the probability distributions over $\mathscr{C}$ they generate. For the purpose of this paper it is more convenient to keep the notations with the state space also for decision under risk. The general case, where a probability measure $P$ on $(S, \Sigma)$ need not be given, is called decision under uncertainty. Thus decision under risk is a special case of decision under uncertainty. Under common continuity assumptions, the sure-thing principle is equivalent to other formulations of 'independence' conditions in the context of risk. This has been elaborated in Fishburn and Wakker (1992).

We assume without further mention that, for decision under risk, stochastic dominance holds, i.e. $f \succeq g$ whenever, for each $\alpha \in \mathscr{C}, P(f(s) \geq \alpha) \geq P(g(s) \geq$ $\alpha$ ). Wakker (1990) discussed in detail the relation between decision under risk and under uncertaint. It was proved there for decision under risk that, under RDU and 
given stochastic dominance, there exists a nondecreasing function $\varphi:[0,1] \rightarrow[0,1]$ such that $W=\varphi \circ P$. The special case where $\varphi$ is the identity function is EU.

Some comments on terminology are in order. In the literature, the term Choquet expected utility is commonly used instead of rank-dependent utility for decision under uncertainty. The term rank-dependent utility is used for decision under risk. Although Choquet expected utility is the more general of the two, still the union of the theories is usually described as rank-dependent utility. This is similar to the convention, also adopted in this paper, of using the term expected utility to describe the union of subjective expected utility (expected-utility-for-uncertainty) and expected-utility-for-risk. This paper simply uses the terms rank-dependent utility and expected utility both for risk and for uncertainty. We are now ready to formulate, informally, the main result of this paper.

Observation 1. Suppose that rank-dependent utility holds, in accordance with one of the presently existing axiomatizations. Then:

(i) The comonotonic sure-thing principle holds.

(ii) The sure-thing principle holds if and only if expected utility holds.

In the following example an RDU model is presented in which the sure-thing principle is satisfied, but EU is still violated. The example demonstrates that the above observation is not trivial and that the restriction to any "presently existing axiomatization' is essential. The example adapts a famous example of Kraft et al. (1959) from qualitative probability theory to the present decision context. The proofs described are also adaptations of proofs from Kraft et al. Section 5 discusses empirical implications of the example. Examples that, like Example 2, show differences in axiomatizations between finite and infinite models, are presented in Krantz et al. (1971, Section 9.1) and Wakker (1988, Section 7) for the context of additive conjoint measurement.

Example 2. Suppose $S=\left\{s_{1}, \ldots, s_{5}\right\}$ and $\mathscr{C}=\{0,1\}$. Gambles are denoted as fivetuples of 0 's and 1's, e.g. $(1,0,0,1,1)$ assigns 1 to $s_{1}, s_{4}$, and $s_{5}$, and 0 to $s_{2}$ and $s_{3}$. RDU is assumed to hold with $U$ the identity function and the capacity $W$ defined as follows: $W(\emptyset)=0, W\left(s_{1}\right)=1 / 31, W\left(s_{2}\right)=2 / 31, W\left(s_{3}\right)=3 / 31$, $W\left(s_{1}, s_{2}\right)=4 / 31, W\left(s_{1}, s_{3}\right)=5 / 31, W\left(s_{4}\right)=6 / 31, W\left(s_{1}, s_{4}\right)=7 / 31, W\left(s_{2}\right.$, $\left.s_{3}\right)=8 / 31, W\left(s_{5}\right)=9 / 31, W\left(s_{1}, s_{2}, s_{3}\right)=10 / 31, W\left(s_{2}, s_{4}\right)=11 / 31, W\left(s_{3}\right.$, $\left.s_{4}\right)=12 / 31, W\left(s_{1}, s_{5}\right)=13 / 31, W\left(s_{1}, s_{2}, s_{4}\right)=14 / 31, W\left(s_{2}, s_{5}\right)=15 / 31$, $W\left(s_{1}, s_{3}, s_{4}\right)=16 / 31, W\left(s_{3}, s_{5}\right)=17 / 31, W\left(s_{2}, s_{3}, s_{4}\right)=18 / 31, W\left(s_{1}, s_{2}\right.$, $\left.s_{5}\right)=19 / 31, W\left(s_{1}, s_{3}, s_{5}\right)=20 / 31, W\left(s_{4}, s_{5}\right)=21 / 31, W\left(s_{1}, s_{2}, s_{3}, s_{4}\right)=$ $22 / 31, W\left(s_{1}, s_{4}, s_{5}\right)=23 / 31, W\left(s_{2}, s_{3}, s_{5}\right)=24 / 31, W\left(s_{1}, s_{2}, s_{3}, s_{5}\right)=25 / 31$, $W\left(s_{2}, s_{4}, s_{5}\right)=26 / 31, W\left(s_{3}, s_{4}, s_{5}\right)=27 / 31, W\left(s_{1}, s_{2}, s_{4}, s_{5}\right)=28 / 31, W\left(s_{1}\right.$, $\left.s_{3}, s_{4}, s_{5}\right)=29 / 31, W\left(s_{2}, s_{3}, s_{4}, s_{5}\right)=30 / 31, W(S)=1$.

It will be obvious that $W$ is a capacity, and RDU implies the comonotonic sure-thing principle. Also, the sure-thing principle holds but, unfortunately, I am 
not aware of an elementary proof. The only proof that I can provide is by simply verifying all cases. The principle is as follows: first one drops from the preferences implied by the above assumptions all gambles that assign outcome 1 to $s_{1}$, so that only the gambles remain that assign outcome 0 to $s_{1}$. The ordering between those remaining gambles is not affected if everywhere the outcome 0 for state $s_{1}$ is replaced by outcome 1 . Thus, it follows that preferences are not affected if a common outcome for the first state is replaced by another common outcome. The same thing is similarly demonstrated for the other four states. Finally, if a preference is not affected by replacing any single common outcome, then, by repeated replacements, preference is not affected by replacing any number of common outcomes by other common outcomes. That is, the sure-thing principle holds.

Next, I demonstrate that no EU representation exists. Suppose such a representation would exist, with additive probability measure $P$. The utility can always be taken as above. It can be seen that Savage's P4 condition (guaranteeing existence of a 'more-likely-than' relation) is satisfied in this example, as well as the 'tradeoff consistency' axiom that was used in Wakker and Tversky (1993) to axiomatize expected utility. But a contradiction can still be derived for EU as follows. Consider the following four preferences: $(1,0,1,0,0) \prec(0,0,0,1,0)$, $(1,0,0,1,0) \prec(0,1,1,0,0),(0,0,1,1,0) \prec(1,0,0,0,1),(0,1,0,0,1) \prec(1,0,1,1,0)$.

Under $\mathrm{EU}$, the $\mathrm{EU}$ values of the gambles should satisfy the corresponding inequalities. However, the EU values of the left four gambles sum to the same as the EU values of the right four acts, i.e. both sums are $2 P\left(s_{1}\right)+P\left(s_{2}\right)+2 P\left(s_{3}\right)$ $+2 P\left(s_{4}\right)+P\left(s_{5}\right)$. This gives a contradiction.

We conclude that in this example RDU holds, along with the sure-thing principle (and also Savage's P4), but no EU representation exists.

The above example considered a finite structure. In all presently existing derivations of RDU, infinite structures have been used, with a continuity assumption that either concerns the outcome space or the state space. The next two sections prove Observation 1 for those two cases.

A sign-dependent generalization of RDU was considered by Starmer and Sugden (1989), Luce and Fishburn (1991), Tversky and Kahneman (1992), and Wakker and Tversky (1993). In these sign-dependent generalizations, the comonotonic sure-thing principle still holds true. It can be proved that strengthening the axiom to the sure-thing principle implies a special case of these theories that was called 'sign-dependent expected utility' in Wakker and Tversky (1993). In words, the theory entails that a person can adopt different capacities for gains than for losses, only now these capacities must both be additive. Wakker and Tversky (1993) pointed out that this theory does not seem to be of interest, i.e. sign-dependence does not seem to be of interest when the capacities must be additive. Therefore, I do not elaborate on the claims of this paragraph. 


\section{The utility range contains an interval}

This section formalizes and proves Observation 1 for the case where the outcome space satisfies a richness condition. It suffices that the range of utility contains an interval. The following theorem is the generic result.

Theorem 3. Suppose that there is a partition of the state space with at least three non-null events, and that $U(\mathscr{C})$ contains a nondegenerate interval. Suppose that rank-dependent utility holds. Then the comonotonic sure-thing principle holds. Expected utility holds if and only if the sure-thing principle holds.

Proof. It is obvious that the comonotonic sure-thing principle holds and that EU implies the sure-thing principle. Hence, we assume the sure-thing principle and derive EU. The result is first proved for a finite state space $\left\{s_{1}, \ldots, s_{n}\right\}$. Let $I \subset U(\mathscr{C})$ be a nondegenerate interval. Consider the set $\mathscr{F}^{\prime}$ of functions from $S$ to $I$ and the binary relation $\succeq^{\prime}$ on $\mathscr{F}^{\prime}$ induced by $\succeq$ on $\mathscr{F}$. If different gambles $g$, $h$ from $\mathscr{F}$ induce the same function $U \circ g=U \circ f=f^{\prime} \in \mathscr{F}^{\prime}$, then $g$ and $h$ are indifferent, so $\succeq^{\prime}$ is well-defined. For simplicity of notation we omit primes and call the functions 'gambles'. Thus we have, in fact, reduced the problem to the case where $\mathscr{E}=I$ and utility is linear.

The preference relation is a weak order that is continuous, i.e. for each gamble $f$ the sets $\{g: g \succeq f\}$ and $\{g: g \preccurlyeq f\}$ are closed. Also, there are at least three non-null states. Hence the sure-thing principle implies that there exists a representation of the form $f \rightarrow \sum_{j=1}^{n} V_{j}\left(x_{j}\right)$ with all $V_{j}$ 's continuous and unique up to scale and location (Debreu, 1960).

Now consider the 'comonotonic cone' $K=\left\{f \in \mathscr{F}: f\left(s_{1}\right) \leq \ldots \leq f\left(s_{n}\right)\right\}$. If a state $s_{j}$ is non-null within this cone then, by the RDU representation, $\succeq$ is strictly increasing with respect to the $j$ th coordinate, so that $V_{j}$ is strictly increasing. If a state $s_{i}$ is null within this cone, then $V_{j}$ must be constant. This shows that a state is non-null if and only if it is non-null within $K$. Hence $K$ has at least three non-null states.

By the uniqueness result in Wakker (1993a, Theorem 3.2) the additive representations provided by RDU and by $\left(V_{j}\right)_{j=1}^{n}$ differ only by scale and location. (Note here that the values of extreme arguments described in Wakker's, 1993a, theorem can be set equal to the RDU values.) This shows that each $V_{j}$ is affine and can be taken as $p_{j} \times$ identity. Thus the representation $f \mapsto \Sigma_{j=1}^{n} V_{j}\left(x_{j}\right)$ provides an expected utility representation on $\mathscr{F}$, which is a particular form of RDU. By standard uniqueness results for RDU (see, for instance, Wakker, 1989a, or 1989b, Chapter VI) the capacity in an RDU representation is unique. Therefore, the capacity $W$ is the same as the additive probability measure of the expected utility representation $f \mapsto \sum_{j=1}^{n} V_{j}\left(x_{j}\right)$, and the RDU representation is necessarily an EU representation.

The result for general state spaces now follows by restriction to finite partitions. 
Remark 4. Theorem 3 can be extended to the 'algebraic setup,' where the range of utility does not necessarily contain an interval, but satisfies the following solvability condition: if gamble $f$ is identical to gamble $h$ except for one nonnull event, and $f \succeq g \geq h$, then the outcomes of $f$ conditional on the nonnull event can be replaced by one outcome such that indifference with $g$ results.

Proof. Instead of the theorem of Debreu (1960) that was invoked in the above proof, now Theorem 6.13 of Krantz et al. (1971) is used; instead of Wakker (1993a, Theorem 3.2), now Wakker (1991a, Theorem 3 and Corollary 6) is used; and instead of Wakker (1989a,b), Wakker (1991b, Theorem 5) is used. (Here the values of extreme arguments can be set equal to the RDU values.)

Theorem 3 can be applied, for the context of decision under uncertainty, to Schmeidler (1989) ${ }^{1}$ and Wakker (1989a,b, Chapter VI, 1993c). The result of Remark 4 can be applied to Wakker (1991b), Nakamura (1990, 1992), and Chew and Karni (1994).

All characterizations for decision under risk known to the author, with the exception of Nakamura (1992), imply that the range of the utility function is an interval; Nakamura (1992) assumes solvability. Thus, the above result can be applied to the mentioned works. They can also be derived from Theorem 6 below. Alternatively, as pointed out above, the sure-thing principle implies the mixtureindependence condition under common continuity conditions (Fishburn and Wakker, 1992). Then EU follows immediately from classical results. Thus, for risk, the result below can be obtained by elementary means. For completeness sake, and to clarify the empirical demarcation between EU and RDU, we have nevertheless incorporated the case of risk in the corollary below. The main motivation for this analysis is not the mathematical results by themselves, but rather the demonstration of what is at the heart of the rank-dependent generalization of EU, and thus how the rank-dependent generalization can be appreciated and tested.

Corollary 5. Rank-dependent utility with the sure-thing principle reduces to expected utility, in the following works.

Decision under uncertainty: Schmeidler (1989); Wakker (1989a,b, 1991b, 1993c); Nakamura (1990, 1992); Chateauneuf (1991); Chew and Karni (1994).

Decision under risk: Quiggin (1982); Yaari (1987); Allais (1988); Chew (1989); Segal (1989, 1990, 1993a,b); Chew and Epstein (1989); Wakker (1991b, 1993c, 1994); Chateauneuf (1990, 1991); and Nakamura (1990, 1992).

\footnotetext{
${ }^{1}$ Schmeidler (1989) assumes that outcomes are probability distributions over prizes. These can be mixed and so can their utility values, implying that the utility range is an interval.
} 
Quiggin (1982) was supplemented in Quiggin and Wakker (1994); Segal (1989) was criticized in Wakker (1993b) and corrected in Segal (1993a); Chew and Epstein (1989) was supplemented in Chew et al. (1993). The rank-dependent theory developed in Luce (1988) deviates in many respects from the classical paradigm. For one thing, it explicitly involves higher-order gambles and a joint receipt operation on gambles. When the theory is modified to fit into the paradigm of this paper, i.e. when attention is restricted to first-order gambles, then the result of Remark 4 can be invoked, because Luce uses solvability axioms as technical conditions. Similarly, the above result can be applied to the multi-stage setups of Segal $(1990,1993 b)$.

\section{Richness of the events}

Savage (1954) derived his expected utility model by imposing a richness assumption on the state space. His result was adapted to RDU by Gilboa (1987). For decision under risk, where all probabilities are assumed available, the richness condition for the state space adopted below is satisfied for RDU as soon as the probability transformation function is continuous. This holds for all contributions in the literature, except Wakker (1994) and Nakamura (1992). The latter were dealt with in Corollary 5 above. The richness condition requires that the capacity be solvable, i.e. for every $A \subset C$ and every $\beta$ between $W(A)$ and $W(C)$ there exists $A \subset B \subset C$ such that $W(B)=\beta$. (This condition was introduced by Gilboa, 1987, who used the term 'convex-ranged'.)

Theorem 6. Suppose that rank-dependent utility holds, with $W$ solvable. Then the comonotonic sure-thing principle holds. W can be taken additive if and only if $\succeq$ satisfies the sure-thing principle.

Proof. Suppose that the sure-thing principle holds. We derive additivity of $W$. (The other implications in the theorem are straightforward.) We only use RDU on the simple gambles. If $\mathscr{E}$ contains only one indifference class then $U$ is constant, $\geq$ is trivial, and so is the theorem.

If $\mathscr{C}$ contains exactly two indifference classes, then we are in fact dealing with comparative probability theory. That is, we define $\geq$ over events by $A \succeq B$ whenever a gamble assigning a better outcome to $A$ and a worse outcome to $S \backslash A$ is weakly preferred to the same gamble with $B$ instead of $A$. This ordering of events is represented by the capacity $W$, hence Savage's P4 axiom (consistency of this ordering of events) follows. The sure-thing principle is Savage's P2 condition. From the sure-thing principle it follows that an event is null if and only if it is null within each maximal comonotonic set, and now Savage's P3 (monotonicity in outcomes) is easily derived from RDU. P5 (nontriviality) follows from the existence of two nonindifferent outcomes and P6 (fineness of events), finally, 
follows from solvability. We have established all conditions that were used in Savage (1954) to obtain an additive personal probability to represent the comparative probability relation over events (for that, his P7 axiom was not used). ${ }^{2}$ Assigning a utility of one to the highest outcome indifference class and a utility of zero to the lowest indifference class, now gives an EU representation for preferences over gambles.

In the remainder of the proof we assume that there are at least three nonindifferent outcomes, say $\alpha \succ \beta \succ \gamma$. Only these three outcomes will be used in the derivation of additivity of $W$. Say $U(\alpha)=1, U(\beta)=0, U(\gamma)=-\tau$ for some $\tau>0$. For simplicity of notation, outcomes are identified with utilities.

For additivity of $W$ we must show that the 'decision weight' that an event $B$ contributes to a disjoint event $A$, i.e. $W(A \cup B)-W(A)$, is independent of $A$, so is identical to $W(B)$ :

$$
W(A \cup B)-W(A)=W(B) .
$$

We use notations such as $(S \backslash B \rightarrow \beta, B \rightarrow \alpha)$ to denote the gamble that assigns outcome $\alpha$ to event $B$ and $\beta$ to event $S \backslash B$. Furthermore we use the notation $\lambda(C, D)=W(C \cup D)-W(D)$; implicit in this notation is that $C$ and $D$ are disjoint. $\lambda(C, D)$ is the decision weight of event $C$ in the rank-dependent formula if event $D$ yields better outcomes than event $C$ and event $S \backslash(C \cup D)$ yields worse outcomes. For the proof of Eq. (3) we distinguish a number of exhaustive (not mutually exclusive) cases.

Case 1. $W(B)=0$ or $\lambda(B, A)=0$ or $W(A)=0$ or $\lambda(A, B)=0$.

Because of symmetry in $A$ and $B$, we need only consider the cases $W(B)=0$ and $\lambda(B, A)=0 . W(B)=0$ if and only if $(S \backslash B \rightarrow \beta, B \rightarrow \alpha) \sim(S \backslash B \rightarrow \beta$, $B \rightarrow \beta$ ). The indifference holds, by the sure-thing principle, if and only if

$$
\begin{aligned}
& \left(S \backslash\left(A^{\prime} \cup B\right) \rightarrow \beta, B \rightarrow \alpha, A^{\prime} \rightarrow \alpha\right) \sim\left(S \backslash\left(A^{\prime} \cup B\right) \rightarrow \beta,\right. \\
& \left.B \rightarrow \beta, A^{\prime} \rightarrow \alpha\right),
\end{aligned}
$$

for all $A^{\prime}$ disjoint from $B$. Equivalent to this indifference is the equality

$$
\lambda\left(B, A^{\prime}\right)=0,
$$

for all $A^{\prime}$ disjoint from $B$. By the sure-thing principle, the above indifference holds for all $A^{\prime}$ disjoint from $B$ if and only if it holds for some, instead of all, $A^{\prime}$ disjoint from $B$. That is equivalent to Eq. (4) for some, instead of all, $A^{\prime}$ disjoint from $B$.

Summarizing, if $W(B)=0$, then Eq. (4) holds for all $A^{\prime}$ disjoint from $B$, in particular for $A$, hence $W(A \cup B)-W(A)=0$. Conversely, if $W(A \cup B)-$

\footnotetext{
${ }^{2}$ For some minor mathematical corrections, see Wakker (1981).
} 
$W(A)=0$, then (4) holds for $A^{\prime}=A$, which is equivalent to $W(B)=0$. Eq. (3) has been established for Case 1 .

Case 2. $W(B)=1$ or $\lambda(B, A)=1$ or $W(A)=1$ or $\lambda(A, B)=1$.

Because of symmetry in $A$ and $B$, we need only consider the cases $W(B)=1$ or $\lambda(B, A)=1$. Using the result as already established, the following statements are equivalent: $W(B)=1, \lambda(S \backslash B, B)=0, W(S \backslash B)=0, W\left(A^{\prime}\right)=0$ for all $A^{\prime}$ disjoint from $B, \lambda\left(B, A^{\prime}\right)=1$ for all $A^{\prime}$ disjoint from $B, \lambda\left(B, A^{\prime}\right)=1$ for some $A^{\prime}$ disjoint from $B$. Here the last two statements at first imply that $W\left(A^{\prime}\right)=0$, next that $W\left(A^{\prime} \cup B\right)=W(B)$, etc.

Case 3. $W(A \cup B)<1$.

We derive the following, seemingly stronger, analogue of Eq. (3).

$$
\lambda(D, C)=W(D),
$$

for all subsets $C, D$ of $A \cup B$ that are disjoint.

Case 3a. $W(D) \leq(1-W(A \cup B)) \tau$, i.e. $W(A \cup B) \leq 1-W(D) / \tau$.

By solvability we can take $E$ such that $S \backslash E \supset(A \cup B)$ and $W(S \backslash E)=1-$ $W(D) / \tau$, i.e. $W(D)=(1-W(S \backslash E)) \tau=\lambda(E, S \backslash E) \tau$. Write $R:=S \backslash(C \cup D \cup$ $E)$. Substitution of RDU gives

$$
(E \rightarrow-\tau, C \rightarrow 0, R \rightarrow 0, D \rightarrow 1) \sim 0 .
$$

By the sure-thing principle also

$$
(E \rightarrow-\tau, R \rightarrow 0, . D \rightarrow 1, C \rightarrow 1) \sim(E \rightarrow 0, R \rightarrow 0, D \rightarrow 0, C \rightarrow 1) .
$$

The last indifference implies, by RDU, that $\lambda(D, C)=\lambda(E, S \backslash E) \tau$. The latter is equal to $W(D)$ as we saw above, hence (5) follows.

The following lemma serves as a preparation for Case $3 \mathrm{~b}$.

Lemma 7. If $\lambda(D, C) \leq(1-W(A \cup B)) \tau$, then (5) holds.

Proof. The inequality in the lemma can be rewritten as $W(A \cup B) \leq 1-$ $\lambda(D, C) / \tau$. By solvability, $E$ can be found such that $S \backslash E \supset(A \cup B)$ and $W(S \backslash E)=1-\lambda(D, C) / \tau$, i.e. $\lambda(D, C)=(1-W(S \backslash E)) \tau$. This implies the indifference in (7) and thus, by the sure-thing principle, (6). This implies again $W(D)=(1-W(S \backslash E)) \tau=\lambda(D, C)$, so that (5) follows. 
Case $3 b . W(D)>(1-W(A \cup B))_{\tau}$.

Now $D$ can, by repeated application of solvability, be partitioned as $D_{1}$ $\cup \ldots \cup D_{n}$, where for each $j, \lambda\left(D_{j}, D_{1} \cup \ldots \cup D_{j-1}\right) \leq(1-W(A \cup B)) \tau$. Here, we use positivity of the right-hand side, which is guaranteed in Case 3. By Lemma $7, \lambda\left(D_{j}, D_{1} \cup \ldots \cup D_{j-1}\right)=W\left(D_{j}\right)$ for each $j$, hence $W\left(D_{j}\right) \leq(1-W(A \cup B)) \tau$ for each $j$. Consequently, by Case 3 a we can apply (5) to $D_{j}$ to establish the second and third equalities in $W(D)=\sum_{j=1}^{n} \lambda\left(D_{j}, D_{1} \cup \ldots \cup D_{j-1}\right)=$ $\sum_{j=1}^{n} W\left(D_{j}\right)=\sum_{j=1}^{n} \lambda\left(D_{j}, C \cup D_{1} \cup \ldots \cup D_{j-1}\right)=\lambda(D, C)$. Again (5) follows.

For Case 3, (5) has been proved, and thus (3).

The extension of Case 3 to the case in which $W(A \cup B)=1$ is nontrivial. One can establish $W(A \cup B) \leq W(A)+W(B)$ by limit-taking. This will, however, not be used below.

The following lemma, a sort of dual to Case 3, serves as preparation for Case 4.

Lemma 8. If $W(A)>0$, then $\lambda(B, A)=\lambda(B, S \backslash B)$.

Proof. It will be shown, seemingly stronger, that

$$
\lambda(D, A \cup C)=\lambda(D, S \backslash D),
$$

for all disjoint $C, D \subset S \backslash A$.

Case (a). $\lambda(D, A \cup C) \leq W(A) / \tau$.

By solvability we can take $E \subset A$ with $\lambda(D, A \cup C) \tau=W(E)$. Set $R:=S \backslash$ $(A \cup C \cup D)$. Then

$$
\begin{aligned}
(R & \rightarrow-\tau, D \rightarrow-\tau, C \rightarrow 0, A \backslash E \rightarrow 0, E \rightarrow 1) \\
& \sim(R \rightarrow-\tau, D \rightarrow 0, C \rightarrow 0, A \backslash E \rightarrow 0, E \rightarrow 0) .
\end{aligned}
$$

By the sure-thing principle we get

$$
\begin{aligned}
(D & \rightarrow-\tau, R \rightarrow 0, C \rightarrow 0, A \backslash E \rightarrow 0, E \rightarrow 1) \\
& \sim(D \rightarrow 0, R \rightarrow 0, C \rightarrow 0, A \backslash E \rightarrow 0, E \rightarrow 0) .
\end{aligned}
$$

This implies $\lambda(D, S \backslash D) \tau=W(E)$. Now (8) follows.

As a preparation for Case(b) in the proof of Lemma 8 , we derive a sort of dual of Case (a):

$$
\lambda(D, S \backslash D) \leq W(A) / \tau \Rightarrow(8) \text { holds. }
$$

Here, by solvability, we can take an event $E \subset A$ such that $W(E)=\lambda(D$, $S \backslash D)_{\tau}$. Then (10) holds, and, by the sure-thing principle, (9). Hence $\lambda(D, A \cup$ $C) \tau=W(E)=\lambda(D, S \backslash D) \tau$ holds true. Now (8), thus (11), follows. 
Case (b). $\lambda(D, A \cup C)>W(A) / \tau$.

Now $D$ can, by repeated application of solvability, be partitioned as $D_{1}$ $\cup \ldots \cup D_{n}$, where for each $\mathrm{j}, \lambda\left(D_{j}, A \cup C \cup D_{1} \cup \ldots \cup D_{j-1}\right) \leq W(A) / \tau$; here we use positivity of $W(A)$, which is assumed in this lemma. We can apply Case (a) to $D_{j}$ (in the role of $D$ ) and $C \cup D_{1} \cup \ldots \cup D_{j-1}$ (in the role of $C$ ), to obtain $\lambda\left(D_{j}, A \cup C \cup D_{1} \cup \ldots \cup D_{j-1}\right)=\lambda\left(D_{j}, S \backslash D_{j}\right)$ for each $D_{j}$. This implies that the antecedent of (11) holds for each $D_{j}$. Consequently, we can apply (8) to each $D_{j}$ (and $C \cup D_{1} \cup \ldots \cup D_{j-1}$ or $S \backslash\left(A \cup D_{1} \cup \ldots \cup D_{j}\right)$, respectively, in the role of $C)$ to establish the second and third equality in $\lambda(D, A \cup C)=\sum_{j=1}^{n} \lambda\left(D_{j}, A\right.$ $\left.\cup C \cup D_{1} \cup \ldots \cup D_{j-1}\right)=\sum_{j=1}^{n} \lambda\left(D_{j}, S \backslash D_{j}\right)=\sum_{j=1}^{\mathrm{n}} \lambda\left(D_{j}, S \backslash\left(D_{1} \cup \ldots \cup D_{j}\right)\right)$ $=\lambda(D, S \backslash D)$. This completes the proof of Lemma 8 .

Case 4. $W(A \cup B)=1,0<W(B)<1$, and $0<W(A)<1$.

By solvability we may assume that $A$ can be partitioned into $A_{1}, A_{2}$, both with positive $W$ value. We get $W(B)=\lambda\left(B, A_{1}\right)=\lambda(B, S \backslash B)=\lambda(B, A)$ : the first equality follows from Case 3 because $W\left(A_{2}\right)>0$ which, by Case 1, implies $\lambda\left(A_{2}, B \cup A_{1}\right)>0$ and hence $W\left(B \cup A_{1}\right)<1$. The second and third equality follow from Lemma 8 because $W\left(A_{1}\right)$ and $W(A)$ are positive.

This completes the proof of (3), and thus of Theorem 6.

Under a natural assumption, the above result applies also to the derivation of RDU in Sarin and Wakker (1992, Theorem 3.1). They assume that a subset of gambles is available on which Savage's (1954) EU axioms are satisfied. The events that are used to generate those gambles are called 'unambiguous' and are, for example, generated by a roulette wheel. Then, by a cumulative dominance axiom, the representation is extended to an RDU representation over all gambles. It is natural to assume that the unambiguous event set is sufficiently rich to imply, for all disjoint (possibly ambiguous) events $A, C$, and $\beta$ such that $W(A)<\beta<$ $W(A \cup C)$, the existence of an unambiguous event $B$ such that $W(A \cup(C \cap B))$ $=\beta$. This condition implies solvability and thus Theorem 6 can be invoked. Theorem 6 cannot be invoked for the more general approach suggested in Sarin and Wakker's Remark 3.3 where the set of conceivable events is no longer assumed to be closed under intersection-taking. This shows once more that a richness assumption is essential for Theorem 3 and that the theorem is not elementary.

\section{Conclusion and discussion}

It has been proved that the critical empirical distinction between expected utility and its rank-dependent generalizations is provided by the distinction between the sure-thing principle and its comonotonic weakening. This at least holds 
true for all presently existing axiomatizations. We have also seen that the continuity assumptions, used in those axiomatizations, are essential, and an example has shown that the result does not hold in general.

I finally discuss an implication of the above results for the empirical interpretation of structural assumptions such as continuity. Arguments in favor of continuity assumptions have been advanced in the literature (Savage, 1954, p. 77). Arrow (1971, p. 48) writes, on a 'Monotone Continuity' axiom that imposes continuity on the state space: 'The assumption of Monotone Continuity seems, I believe correctly, to be the harmless simplification almust inevitable in the formalization of any real-life problem.' The example has, however, demonstrated a limitation to the empirical meaning of the conventional axiomatizations that adopt continuity assumptions. Let me explain the point in more detail: a person who would gather the data as described in the example and who would not be aware of the limitations of the continuity axioms, might erroneously believe that the data must conform to expected utility. After all, the sure-thing principle, and actually all of Savage's (1954) intuitive axioms, as well as the intuitive expected utility axioms of Wakker and Tversky (1993), are satisfied. In addition, continuity conditions can never be falsified by finite data. Still, as we have demonstrated, expected utility cannot hold in the example. Apparently, while the intuitive axioms are not violated by the data and can be preserved in extensions to infinite models, and also the continuity axioms are not violated by the data and can also be preserved in extensions to infinite models, it is impossible to extend the data to an infinite model in which the intuitive and continuity axioms are jointly satisfied.

\section{References}

Allais, M., 1988, The general theory of random choices in relation to the invariant cardinal utility function and the specific probability function, In: B.R. Munier, ed., Risk, decision and rationality (Reidel, Dordrecht), 233-289.

Arrow, K.J., 1971, Essays in the thcory of risk-bcaring (North-Holland, Amsterdam).

Chateauneuf, A., 1990, On the use of comonotonicity in the axiomatization of EURDP theory for arbitrary consequences, CERMSEM, University of Paris I; extended abstract presented at Fifth International Conference on the Foundations and Applications of Utility, Risk and Decision Theory (FUR-90).

Chateauneuf, A., 1991, On the use of capacities in modeling uncertainty aversion and risk aversion, Journal of Mathematical Economics 20, 343-369.

Chew, S.H., 1989, The rank-dependent quasilinear mean, Unpublished manuscript, Department of Economics, University of California, Irvine, USA.

Chew, S.H. and L.G. Epstein, 1989, A unifying approach to axiomatic non-expected utility theories, Journal of Economic Theory 49, 207-240.

Chew, S.H., L.G. Epstein and P.P. Wakker, 1993, A unifying approach to axiomatic non-expected utility theories: Corrigenda, Journal of Economic Theory 59, 183-188.

Chew, S.H. and E. Karni, 1994, Choquet expected utility with a finite state space: Commutativity and act-independence, Journal of Economic Theory 62, 469-479. 
Chew, S.H. and P.P. Wakker, 1993, Generalizing Choquet expected utility by weakening Savage's sure-thing principle, University of California, Irvine Research Unit in Mathematical Behavioral Sciences, Irvine CA, USA.

Debreu, G., 1960, Topological methods in cardinal utility theory, In: K.J. Arrow, S. Karlin and P. Suppes, eds., 1959, Mathematical methods in the Social Sciences (Stanford University Press, Stanford, CA), 16-26.

Fennema, H. and P.P. Wakker, 1995, A Test of Rank Dependent Utility in the Context of Ambiguity (NICI, University of Nijmegen, Nijmegen).

Fishburn, P.C. and P.P. Wakker, 1992, The invention of the independence condition for preferences, Management Science, in press.

Gilboa, 1., 1987, Expected utility with purely subjective non-additive probabilities, Journal of Mathematical Economics 16, 65-88.

Kraft, C.H., J. Pratt and A. Seidenberg, 1959, Intuitive probability on finite sets, Annals of Mathematical Statistics 30, 408-419.

Krantz, D.H., R.D. Luce, P. Suppes and A. Tversky, 1971, Foundations of measurement, Vol. 1. (Additive and Polynomial representations) (Academic Press, New York).

Luce, R.D., 1988, Rank-dependent, subjective expected-utility representations, Journal of Risk and Uncertainty 1, 305-332.

Luce, R.D. and P.C. Fishburn, 1991, Rank- and-sign dependent linear utility models for finite first-order gambles, Journal of Risk and Uncertainty 4, 29-59.

Nakamura, Y., 1990, Subjective expected utility with non-additive probabilities on finite state spaces, Journal of Economic Theory 51, 346-366.

Nakamura, Y., 1992, Multi-symmetric structures and non-expected utility, Journal of Mathematical Psychology 36, 375-395.

Quiggin, J., 1982, A theory of anticipated utility, Journal of Economic Behaviour and Organization 3, 323-343.

Quiggin, J. and P.P. Wakker, 1994, The axiomatic basis of anticipated utility: A clarification, Journal of Economic Theory 64, 486-499.

Sarin, R.K. and P.P. Wakker, 1992, A simple axiomatization of nonadditive expected utility, Econometrica $60,1255-1272$.

Savage, L.J., 1954, The foundations of statistics (Wiley, New York). (Second edition 1972, Duver, New York.)

Schmeidler, D., 1989, Subjective probability and expected utility without additivity, Econometrica 57, 571-587.

Segal, U., 1989, Anticipated utility: A measure representation approach, Annals of Operations Research 19, 359-373.

Segal, U., 1990, Two-stage lotteries without the reduction axiom, Econometrica 58, 349-377.

Segal, U., 1993a, The measure representation: A correction, Journal of Risk and Uncertainty 6, 99-107.

Segal, U., 1993b, Order indifference and rank-dependent probabilities, Journal of Mathematical Economics 22, 373-397.

Starmer, C. and R. Sugden, 1989, Violations of the independence axiom in common ratio problems: An experimental test of some competing hypotheses, Annals of Operations Research 19, 79-101.

Tversky, A. and D. Kahneman, 1992, Advances in prospect theory: Cumulative representation of uncertainty, Journal of Risk and Uncertainty 5, 297-323.

Wakker, P.P., 1981, Agreeing probability measures for comparative probability structures, The Annals of Statistics 9, 658-662.

Wakker, P.P., 1988, The algebraic versus the topological approach to additive representations, Journal of Mathematical Psychology 32, 421-435.

Wakker, P.P., 1989a, Continuous subjective expected utility with nonadditive probabilities, Journal of Mathematical Economics 18, 1-27. 
Wakker, P.P., 1989b, Additive representations of preferences, a new foundation of decision analysis (Kluwer Academic Publishers, Dordrecht).

Wakker, P.P., 1990, Under stochastic dominance Choquet-expected utility and anticipated utility are identical, Theory and Decision 29, 119-132.

Wakker, P.P., 1991a, Additive representations on rank-ordered sets. I. The algebraic approach, Journal of Mathematical Psychology 35, 501-531.

Wakker, P.P., 1991b, Additive representations of preferences, a new foundation of decision analysis: The algebraic approach, in: J.P. Doignon and J.C. Falmagne, eds., Mathematical psychology: Current developments (Springer Verlag, Berlin), 71-87.

Wakker, P.P., 1993a, Additive representations on rank-ordered sets II. The topological approach, Journal of Mathematical Economics 22, 1-26.

Wakker, P.P., 1993b, Counterexamples to Segal's measure representation theorem, Journal of Risk and Uncertainty 6, 91-98.

Wakker, P.P., 1993c, Unbounded utility for Savage's 'Foundations of Statistics', and other models, Mathematics of Operations Research 18, 446-485.

Wakker, P.P., 1994, Separating marginal utility and probabilistic risk aversion, Theory and Decision 36, $1-44$.

Wakker, P.P., I. Erev and E. Weber, 1994, Comonotonic independence: The critical test between classical and rank-dependent utility theories, Journal of Risk and Uncertainty 9, 195-230.

Wakker, P.P. and A. Tversky, 1993, An axiomatization of cumulative prospect theory, Journal of Risk and Uncertainty 7, 147-176.

Yaari, M.E., 1987, The dual theory of choice under risk, Econometrica 55, 95-115. 